\title{
GYROSTAT MODEL REGULAR AND CHAOTIC BEHAVIOR*
}

\author{
Svetoslav Nikolov \\ Institute of Mechanics, Bulgarian Academy of Science, \\ Acad. G. Bonchev St., Bl. 4, 1113 Sofia, Bulgaria, \\ University of Transport, 158, G. Milev St., 1574 Sofia, Bulgaria, \\ e-mail: S.Nikolov@imbm.bas.bg \\ NATALIYA NEDKOVA \\ University of Transport, 158, G. Milev St., 1574 Sofia, Bulgaria, \\ e-mail: Nataliya_Nedkova@abv.bg
}

[Received 09 September 2015. Accepted 16 November 2015]

\begin{abstract}
During recent years, the interest in the phenomena of chaos in gyroscopic systems has been increasing. It is well-known, that depending on the speed of rotation, a gyroscopic system may lose or gain stability. Despite the overwhelming number of studies reporting the occurrence of various chaotic structures, little is known yet about the construction details and the generality of the underlying bifurcation scenarios that give rise to such chaotic (complex) behaviour.

In this paper, we report a detailed investigation of the abundance of regular and chaotic behaviour for rigid body (gyrostat) motion. The model contains 6 parameters that may be tuned to produce rich dynamical scenarios. The results confirm that homoclinic and heteroclinic structures with two fixed points from saddle-focus type occur and the emergence of Shilnikov chaos takes place. Finally, we find new results concerning the system's evolution and bifurcation scenarios for its routes to chaos.

KEY WORDS: Dynamical systems, chaos, gyrostat, homoclinic and heteroclinic orbits.
\end{abstract}

\section{Introduction}

Modelling is a powerful tool in the processes simulation in physics and technologies, dealing with different time and spatial scales, and in the mechan-

\footnotetext{
${ }^{*}$ Corresponding author e-mail: S.Nikolov@imbm.bas.bg

This paper is funded by the grant No 1616/22.04.2014 of the University of Transport "T. Kableshkov" Sofia, Bulgaria.
} 
ical characterization of system parameters. It is also effective in the interpretation and design of experiments, as well as in the new effects and phenomena prediction. It is clear, that modelling will play an increasing role in improving our understanding of the physical processes in mechanical systems, under normal and abnormal conditions.

In an array of great discoveries in the twentieth century, three of them certainly belong to physics: 1) the theory of relativity of Albert Einstein (without ignoring the great merit of Henri Poincaré); 2) quantum mechanics, associated with a large number of scientists from different countries and 3) chaos theory, associated mostly with the name of the American theorist Edward Lorenz, a meteorologist. Again, Henri Poincaré has a contribution to its development, and later many scholars, the list of whose names cannot fit into several dozen pages. It will be only mentioned that the first to use the word chaos in its modern understanding in science are Li and Yorke in 1975 [1], and that the authors of the concept of the strange attractor are Ruelle and Takens in 1971 [2].

There has been a large amount of recent interest in the investigation of gyroscope dynamics. The gyroscope has attributes of great utility to navigational and aeronautical engineering, biology, optics, etc. [3-8]. Different types of gyroscopes (with linear or nonlinear damping, fluid, etc.) are investigated for predicting dynamic responses such as regular and chaotic motions [9-11].

Dissipative systems are a special class of dynamical systems. In general, dissipative mechanical systems in more than two dimensions have bounded and unbounded orbits, depending on the energy. The bounded trajectories of these systems do not converge to an equilibrium point or to a periodic or quasiperiodic orbit. In this case, the flow is essentially aperiodic. A dynamical system is dissipative, if its phase volume contracts continuously, i.e. $D_{i}<0$ $(i=1, \ldots, n)$. Only dissipative dynamical systems have attractors $[12,13]$.

Attractors are the adequate mathematical (geometrical) representations of time order and chaos that can be: stable equilibrium, stable periodic motions (auto-waves) or strange attractors. Mathematical representations of spatial order and chaos are saddle equilibrium, saddle periodic movements or complex saddle invariant sets.

An important step towards the understanding of the global dynamics of a system of differential equations is the analysis of the existence of homoclinic/heteroclinic orbits (cycles). It is well-known, that a heteroclinic cycle is a sequence of trajectories connecting a set of fixed points in a topological circle. A classical heteroclinic cycle is a loop that consists of saddle equilibrium states connected to one another by their separatrices. The special case of a cycle consisting of one trajectory and one fixed point is usually called a homoclinic 
trajectory $[14,15]$.

A homoclinic trajectory $f(x, t)$, (or a homoclinic (separatrix) loop) is such that the 'inset' to a fixed point of an attractor, $x_{0}$, is the same as the 'outset' from the same point. It obeys the rule that $f(x, t) \rightarrow x_{0}$ for $t \rightarrow \infty$, $t \rightarrow-\infty$ [16]. According to Peixoto's theorem [17, 18], homoclinic bifurcations are structurally unstable and are therefore, destroyed by small perturbations. Consequently, they are more difficult to identify than a local bifurcation, because knowledge of the global properties of the phase space trajectories is required. A systematic characterization of homoclinicity around a saddle-focus equilibrium was provided by Shilnikov [19-21]. In this scenario, reinjection occurs along a well-defined vector associated with a real system eigenvalues, with ejection from the vicinity of the equilibrium, subsequently effected on a spiral path located on a transverse plane. A necessary condition for this mechanism is that the saddle-focus index is $\delta=\left|\operatorname{Re}\left(\chi_{2} / \chi_{1}\right)\right|<1$, where $\chi_{1}$ and $\chi_{2}$ are the leading eigenvalues. Here, we note that $\chi_{1}$ is determining the rate of approaching and $\chi_{2}$ is determining the rate of leaving the stable point. If thus Shilnikov condition is satisfied, an infinite number of nonperiodic trajectories coexist in the vicinity of a homoclinic trajectory bi-asymptotic to the saddle-focus.

A nice example is the Lorenz system [22], which has an important historical relevance in the development of chaos theory. Now, this system is considered as a paradigmatic example of a chaotic system [23]. This system is adaptive, if $\nabla \dot{x}_{i}$ takes both positive and negative values. Rigid bodies are bodies that cannot deform and change their shape, but they can translate and rotate. In $[24,25]$, the authors remark that the Lorenz system admits a purely mechanical model of two rigid bodies - lifting and lifted axi-symmetric rotors. The system is firstly suggested by Gluhovsky in [26] and has the following property: when the rotor moves relative to the lifting body, then the distribution mass remains unaltered in space. Thus, the system tensor is constant and such a system is called a gyrostat (see Fig. 1). Further, it is assumed that its center of mass is fixed, and that the ellipsoid of inertia has the form of a rotation ellipsoid.

In case of small angular velocities, the motion of the system can be presented from the following differential equation [9, 27]:

$$
\dot{M}=M \times A M+B M,
$$

where $M$ is the vector of kinematical moment about the coordinate system of the rigid body, $A=I^{-1}=\operatorname{diag}\left(a_{1}, a_{2}, a_{3}\right)$ (where $I$ is the inertial tensor) and $\boldsymbol{B}$ is a constant matrix. For the dissipative case, i.e. div $<0$, the condition 


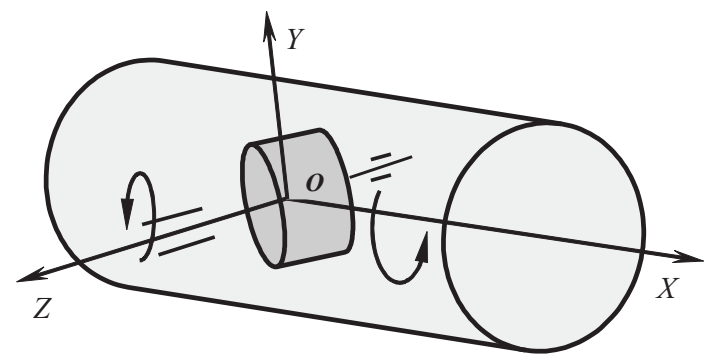

Fig. 1. Two bodies - lifting and lifted axi-symmetric rotor

$\operatorname{Tr} \boldsymbol{B}<0$ is valid. In [28], it is shown for specific values of system parameters, that two attractors have place. It is well known, that system (1) has two particular cases: (i) those of Greenhill and (ii) Klein \& Sommerfeld [29]. The trajectories lie in integral surfaces in these two cases. For some complicated cases, system (1) has two strange attractors.

According to Fig. 1, the system (1) can be written in the form:

$$
\begin{aligned}
& \dot{M}_{1}=c_{1} M_{1}+M_{2}+c_{2} M_{2} M_{3}, \\
& \dot{M}_{2}=-M_{1}-c_{3} M_{2}+c_{4} M_{1} M_{3}, \\
& \dot{M}_{3}=c_{5} M_{3}-c_{6} M_{1} M_{2},
\end{aligned}
$$

where $M_{1}, M_{2}$ and $M_{3}$ are the projections of the instantaneous angular velocity onto the $X$-, $Y$ - and $Z$-axes (see Fig. 1), and $c_{1} \div c_{6}$ are parameters (in form of algebraic complexes), given in [24, 28].

In general, the investigation of such model would start with the computation of steady states. The equilibrium (steady state) points of the system (2) are found by equating the right-hand sides of (2) to zero. Thus, it is easy to see that equilibrium points of the system (2) are:

$$
\mathbf{O}_{1}: \bar{M}_{1}=\bar{M}_{2}=\bar{M}_{3}=0, \quad \text { first fixed point }
$$

(4) $\mathbf{O}_{2,3,4,5}$ :

$$
\bar{M}_{1}=\frac{1}{c_{1}} \bar{M}_{2}\left(1+\frac{c_{2} c_{6}}{c_{1} c_{5}} \frac{\bar{M}_{2}^{2}}{1-\frac{c_{2} c_{6}}{c_{1} c_{5} \bar{M}_{2}^{2}}}\right), \bar{M}_{3}=\frac{c_{6}}{c_{1} c_{5}} \frac{\bar{M}_{2}^{2}}{1-\frac{c_{2} c_{6}}{c_{1} c_{5}} \bar{M}_{2}^{2}},
$$

$$
\bar{M}_{2}^{4}-\frac{1}{c_{2}^{2} c_{3} c_{6}}\left[c_{5}\left(c_{2}+c_{4}\right)+2 c_{1} c_{2} c_{3} c_{5}\right] \bar{M}_{2}^{2}+\frac{c_{1} c_{5}^{2}}{c_{2}^{2} c_{3} c_{6}^{2}}\left(1+c_{1} c_{3}\right)=0 .
$$


For the reason that $\left(c_{2}+c_{4}\right)^{2}+4 c_{1} c_{2} c_{3}\left(c_{1} c_{2} c_{3}+c_{4}\right)>0$, the system (2) has five real fixed points - see Fig. 2 a, b.
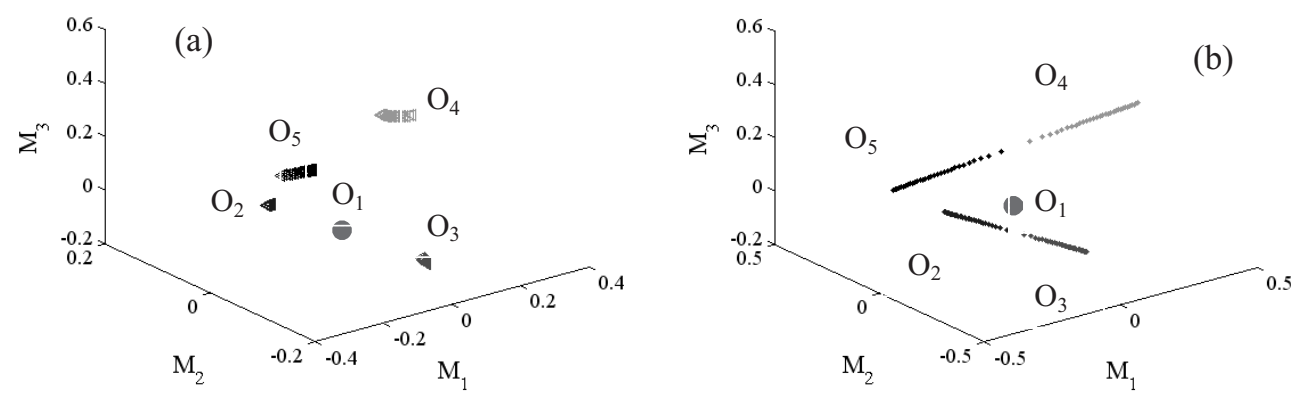

Fig. 2. (a) Fixed points of system (2) in $M_{1} M_{2} M_{3}$ coordinates as a function of $c_{1}$;

(b) fixed points of system (2) in $M_{1} M_{2} M_{3}$ coordinates as a function of $c_{5}$

The system (2) enjoys the natural symmetry $\left(M_{1}, M_{2}, M_{3}\right) \rightarrow\left(-M_{1}\right.$, $\left.-M_{2}, M_{3}\right)$. The $M_{3}$-axis is invariant. All trajectories, which start on the $M_{3^{-}}$ axis remain on it.

The divergence of the flow (2) is:

$$
D_{3}=\frac{\partial \dot{M}_{1}}{\partial M_{1}}+\frac{\partial \dot{M}_{2}}{\partial M_{2}}+\frac{\partial \dot{M}_{3}}{\partial M_{3}}=c_{5}-\left(c_{1}+c_{3}\right) .
$$

The system (2) is dissipative, when $D_{3}<0$, i.e. $c_{5}<c_{1}+c_{3}$. It means, that a volume element $D_{3}\left(t_{0}\right)$ is contracted by the flow into a volume $D_{3}(t)=D_{3}\left(t_{0}\right) \times e^{-\left(c_{1}+c_{3}-c_{5}\right) t}$. That is, as $t \rightarrow+\infty$, each volume containing the system trajectories shrinks to zero at an exponential rate (independently of the system states) $D_{3}=-\left(c_{1}+c_{3}-c_{5}\right)$. Hence, all system trajectories will be confined to a specific subset of zero volume. For example, in 1981 Leipnik and Newton [28] found that for $c_{5}<0.8$, the system is dissipative and all volumes in the phase space must contract uniformly, even though crosssectional and all trajectories except those trapped at the rest points, diverge to infinity. Simulations suggest that for $c_{5}=0.175$ the system (2) has two strange attractors.

The paper is further organized as follows: in Section 2, 3 and 4 we present analytical and numerical results, concerning the system (2) behaviour for different values of bifurcation (control) parameters $c_{1}$ and $c_{5}$. In Section 5 we discuss and summarize our results. 


\section{Theoretical analysis}

In this section, we investigate the system (2), which presents an autonomous nonlinear 3D dynamical model.

Generally, in order to determine the character of fixed points (Eqs. (3) and (4)), we make the following substitutions into (2):

$$
M_{1}=\bar{M}_{1}+w_{1}, \quad M_{2}=\bar{M}_{2}+w_{2}, \quad M_{3}=\bar{M}_{3}+w_{3} .
$$

Hence, after some transformations, the system (2) has the form:

$$
\begin{aligned}
& \dot{w}_{1}=-c_{1} w_{1}+b_{1} w_{2}+b_{2} w_{3}+c_{2} w_{2} w_{3}, \\
& \dot{w}_{2}=-b_{3} w_{1}-c_{3} w_{2}+b_{4} w_{3}+c_{4} w_{1} w_{3}, \\
& \dot{w}_{3}=-b_{5} w_{1}-b_{6} w_{2}+c_{5} w_{3}-c_{6} w_{1} w_{2},
\end{aligned}
$$

where

$$
\begin{aligned}
& b_{1}=1+c_{2} \bar{M}_{3}, \quad b_{2}=c_{2} \bar{M}_{2}, \quad b_{3}=1-c_{4} \bar{M}_{3}, \\
& b_{4}=c_{4} \bar{M}_{1}, \quad b_{5}=c_{6} \bar{M}_{2}, \quad b_{6}=c_{6} \bar{M}_{1} .
\end{aligned}
$$

According to [30], the Routh-Hurwitz conditions for stability of fixed points (3) and (4), can be written in the form:

$$
\begin{aligned}
& p=c_{1}+c_{3}-c_{5}>0, \\
& q=c_{1}\left(c_{3}-c_{5}\right)-c_{3} c_{5}+b_{1} b_{3}+b_{2} b_{5}+b_{4} b_{6}>0, \\
& r=-\left[c_{5}\left(c_{1} c_{3}+b_{1} b_{3}\right)-b_{5}\left(b_{1} b_{4}+b_{2} c_{3}\right)+b_{6}\left(b_{2} b_{3}-b_{4} c_{1}\right)\right]>0, \\
& R=p q-r=\left(c_{3}-c_{5}\right)\left[c_{1}\left(c_{1}+c_{3}-c_{5}\right)-c_{3} c_{5}+b_{4} b_{6}\right]+ \\
& \quad b_{1} b_{3}\left(c_{1}+c_{3}\right)+b_{2} b_{5}\left(c_{1}-c_{5}\right)-b_{1} b_{4} b_{5}+b_{2} b_{3} b_{6}>0 .
\end{aligned}
$$

Here, the notations $p, q, r$ and $R$ are taken from [30]. The characteristic equation of the system (7) (which is equivalent to system (2)), can be written as:

$$
\chi^{3}+p \chi^{2}+q \chi+r=0
$$

Here, we note that, the five fixed points at bifurcation parameters $c_{1}$ and/or $c_{5}$ are always from saddle-focus type (and not from saddle type as is noted in [39] and many another papers) - negative real eigenvalue and complex eigenvalues with positive real part (unstable focus) (see Fig. 3 - left panel), and positive real eigenvalue and complex eigenvalues with negative real part (stable focus) (see Fig. 3 - right panel). These fixed points can be included in homoclinic and heteroclinic structures with two, three, four and five equilibriums of 


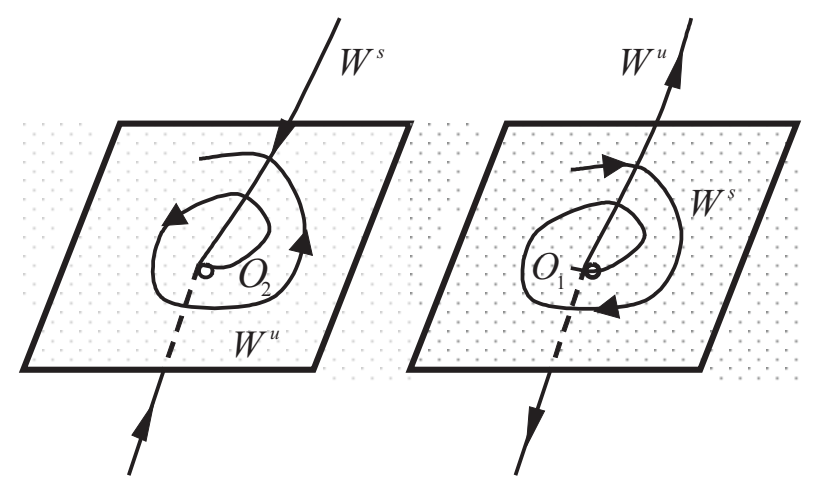

Fig. 3. Fixed points from saddle-focus type of system (2) with: unstable focus (complex eigenvalues with positive real part) - left panel and stable focus (complex eigenvalues with negative real part) - right panel

Shilnikov type, where their invariant manifolds $W^{s}$ and $W^{u}$, are meeting each other in a most intricate manner.

For $c_{1}=c_{3}=0.4, c_{2}=10, c_{4}=c_{6}=5$ and $c_{5}=0.3$ the equilibriums and their eigenvalues are given by:

$$
\begin{array}{ll}
O_{1}=(0,0,0), & \text { then }\left(\chi_{1}, \chi_{2}, \chi_{3}\right)=(0.3,-0.4 \pm i) ; \\
O_{2}=(-0.0413,0.1602,-0.1103), & \text { then }\left(\chi_{1}, \chi_{2}, \chi_{3}\right)=(-0.8,0.15 \pm 1.1419 i) ; \\
O_{3}=(0.0413,-0.1602,-0.1103), & \text { then }\left(\chi_{1}, \chi_{2}, \chi_{3}\right)=(-0.8,0.15 \pm 1.1419 i) ; \\
O_{4}=(0.3129,0.0403,0.2103), & \text { then }\left(\chi_{1}, \chi_{2}, \chi_{3}\right)=(-0.8,0.15 \pm 1.5831 i) ; \\
O_{5}=(-0.3129,-0.0403,0.2103), & \text { then }\left(\chi_{1}, \chi_{2}, \chi_{3}\right)=(-0.8,0.15 \pm 1.5831 i) ;
\end{array}
$$

A heteroclinic cycle is one of the common scenarios of the formation or death of a limit cycle, when the limit cycle emanates from, or approaches the heteroclinic cycle as a singular limit, respectively [15, 31]. There are known cases in which a unique limit cycle is born and certain criteria can be used to determine if this cycle must be stable or unstable. In our case here, the known analytical results are not applicable [32,33], and we are forced to use numerical simulations and specific features of our system. Hence, we find new results for its bifurcation behaviour and routes to chaos.

\section{Shilnikov criteria for chaos}

In Section 2, we obtained that system (2) has fixed points from saddlefocus type. Therefore, in this case the Shilnikov theory for analyzing of bifurcations of homoclinic/heterclinic orbits and existence of chaos in flow in $R^{3}$, 
can be used [19, 34-36]. Below, we explain shortly the general results obtained by Shilnikov.

We focus our considerations on bifurcations from homoclinic and heteroclinic orbits between saddle-foci equilibrium in a three dimensional autonomous system:

$$
\dot{x}=\frac{d x}{d t}=f(x, \mu), \quad x \in R^{3}, \quad \mu \in R^{1},
$$

where the nonlinearity $f$ is sufficiently smooth for the results to hold. The Shilnikov criteria for saddle-focus equilibrium are summarized in the following theorem [36-38]:

Theorem. Suppose that system (11) has at $\mu=0$ a saddle-focus equilibrium point $x_{0}=0$ with eigenvalues $\chi_{1}(0)>0>\operatorname{Re} \chi_{2,3}(0)$ and a homoclinic orbit $\Gamma_{0}$. Assume the following genericity conditions:

$$
\begin{array}{ll}
\left(H_{1}\right) & \sigma_{0}=\chi_{1}(0)+\operatorname{Re} \chi_{2,3}(0)<0 ; \\
\left(H_{2}\right) & \chi_{2}(0) \neq \chi_{3}(0) ; \\
\left(H_{3}\right) & \beta^{\prime}(0) \neq 0, \quad \text { where } \beta(\mu) \text { is the split function; } \\
\left(H_{4}\right) & \sigma_{0}=\chi_{1}(0)+\operatorname{Re} \chi_{2,3}(0)>0 .
\end{array}
$$

Hence, $(i)$ if the conditions $\left(H_{1}-H_{3}\right)$ are valid, then system (11) has a unique and stable limit cycle $L_{\beta}$ in a neighborhood $U_{0}$ of $\Gamma_{0} \cup x_{0}$ for all sufficiently small $\beta>0$; (ii) if the conditions $\mathrm{H}_{2}$ and $\mathrm{H}_{4}$ are valid, then system (11) has an infinite number of saddle limit cycle in a neighborhood $U_{0}$ of $\Gamma_{0} \cup x_{0}$ for all sufficiently small $|\beta|$.

\section{Remarks (about part (i) of Theorem):}

1. For all sufficiently small $\beta \leq 0$, the system (11) has no periodic orbits in $U_{0}$ and the unstable manifold $W^{u}\left(x_{0}\right)$ tends to the cycle $L_{\beta}$;

2. For $\beta=0$, the cycle period tends to infinity.

\section{Remarks (about part (ii) of Theorem):}

1. For $\beta$ taking positive or negative values, an infinite number of bifurcations occur, as some of these bifurcations are related to a "basic" limit cycle;

2. The "basic" cycle disappears and appears via tangent/fold bifurcation infinity many times. Moreover, the cycle also exhibits an infinite number of period-doubling bifurcations; 
3. The "basic" cycle (as well as the secondary cycles generated by perioddoublings) can be stable or repelling, depending on the sign of the divergence of (11) at the saddle-focus, i. e.:

$$
\sigma_{1}=(\operatorname{div} f)\left(x_{0}, 0\right)=\chi_{1}+2 \operatorname{Re} \chi_{2,3} .
$$

If $\sigma_{1}<0$, the "basic" cycle near the bifurcation is stable - there are only short intervals of $\beta$, within which it is stable. If $\sigma_{1}>0$, there are intervals where the "basic" cycle is absolutely unstable (repelling);

4. For $\beta_{i} \rightarrow 0\left(\beta_{i}>0\right)$, the system (11) has double homoclinic orbits with different (increasing) number of rotations near the saddle-focus.

Initially it was assumed that $n_{-}=\operatorname{dim} W^{s}=2$ and $n_{+}=\operatorname{dim} W^{u}=1$. We have to reverse the direction of time, in order to apply the above results in the opposite case $-n_{-}=1, \quad n_{+}=2$, Hence, the following substitutions are valid: $\chi_{j} \rightarrow-\chi_{j}, \quad \sigma_{i} \rightarrow-\sigma_{i}$ and "stable" $\rightarrow$ "repelling".

\section{Numerical analysis}

In the previous two sections, we obtained and showed some analytical results, that we shall use in our numerical analysis in the system (2). According to $[28,39]$, the corresponding values of the dimensionless parameters $c_{1} \div c_{6}$ are:

$$
\begin{array}{lll}
c_{1} \in[0.4,0.9], & c_{2}=10, & c_{3} \in[0.4,0.9], \\
c_{4}=5, & c_{5} \in[0,0.9], & c_{6}=5 .
\end{array}
$$

In order to compare the predictions with the numerical results, the governing equations of system (2) were solved numerically using MATLAB [40]. The initial conditions for all simulations are $(0.349,0,-0.16)$ or $(0.349,0,-0.18)$.

Figure 4 shows the bifurcation diagram for system (2): values of $M_{2}$ coordinate, $\left(M_{2}\right)_{n}$ are plotted against $c_{5}$ regarded as a continuously varying bifurcation (control) parameter. We see that at $c_{5} \in[0.181,0.2]$ the system (2) has chaotic solution - two strange attractors in the regions of two heteroclinic cycles. It is interesting to note that after $c_{5}=0.2$ (till the end of the interval), the inverse period-doubling bifurcations occur and the system passes from chaotic regime to a regular one. For $c_{5} \in[0.28,0.7]$, the system (2) has periodic solution with period three.

We also conclude, that an apparent sudden collapse in the chaotic attractor size occurs at a value of the control parameter $c_{5} \approx 0.149$. Such a sudden qualitative change in a chaotic attractor is known as interior crisis [41, 


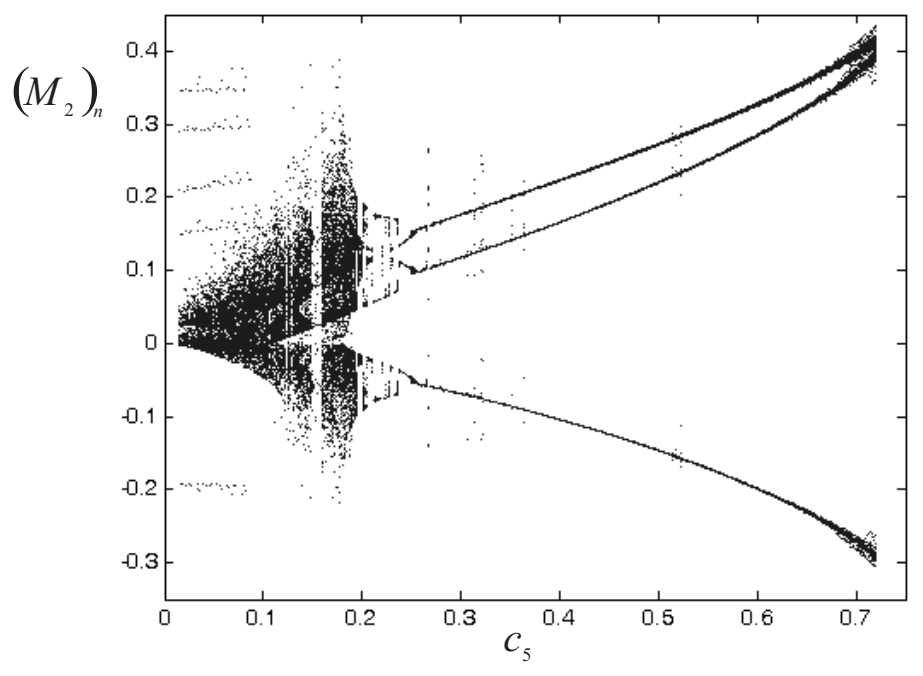

Fig. 4. Bifurcation diagram $\left(M_{2}\right)_{n}$ versus $c_{5}$, generated by computer solutions of the system $(2)$, computed with the parameters: $c_{1}=c_{3}=0.4$, $c_{2}=10, c_{4}=5, c_{5} \in[0.01,0.78], c_{6}=5$

42]. This conclusion is in accordance with the Theorem explained in previous Section 3 and the results in the Appendix-see Figs 7 and 8. For $c_{5} \in[0.15,0.18]$ the system has one homoclinic cycle (for fixed point $O_{1}$ ) and two heteroclinic cycles $-\Gamma_{0}^{2,3} \subset W^{u(s)}\left(O_{2}\right) \cap W^{s(u)}\left(O_{3}\right) ; \Gamma_{0}^{4,5} \subset W^{u(s)}\left(O_{4}\right) \cap W^{s(u)}\left(O_{5}\right)$. For details, see Appendix Fig. 11. From dynamical point of view, as $c_{5}$ increases, the fixed points $\mathrm{O}_{2}-\mathrm{O}_{5}$ change the position (see Fig. 2 a). The saddle-foci $\mathrm{O}_{2}-\mathrm{O}_{5}$ remove from $\mathrm{O}_{1}$ and its unstable manifolds do not influence on the saddle-focus $O_{1}$, i.e. on the stability of the "basic" cycle. This excludes the possibility of a heteroclinic cycle for any finite $c_{5}$ larger than 0.4 .

In Fig. 5, the bifurcation diagram of system (2) (as $\left.c_{1} \in[0.4,0.88]\right)$ is shown. It can be seen, that at $c_{1}>0.42$ chaotic solution with two strange attractors occurs. The white zones, seen in Fig. 6, correspond to inverse bifurcations, which are very fast. At about $c_{5} \in[0.4,0.419]$, a single strange attractor takes place, which at the end of the interval bifurcates into two strange attractors. Comparing results presented in Figs 4, 5 and 6, we conclude that the case, when $c_{1}$ is a bifurcation parameter, is more interesting from dynamical point of view than those with bifurcation parameter $c_{5}$. Discussing the results shown in Fig. 6, it is seen that for $c_{5} \in[0.422,0.428]$ and $c_{5} \in[0.758,0.763]$ the system has periodic solutions and homoclinic cycle (for fixed point $O_{1}$ ) and 


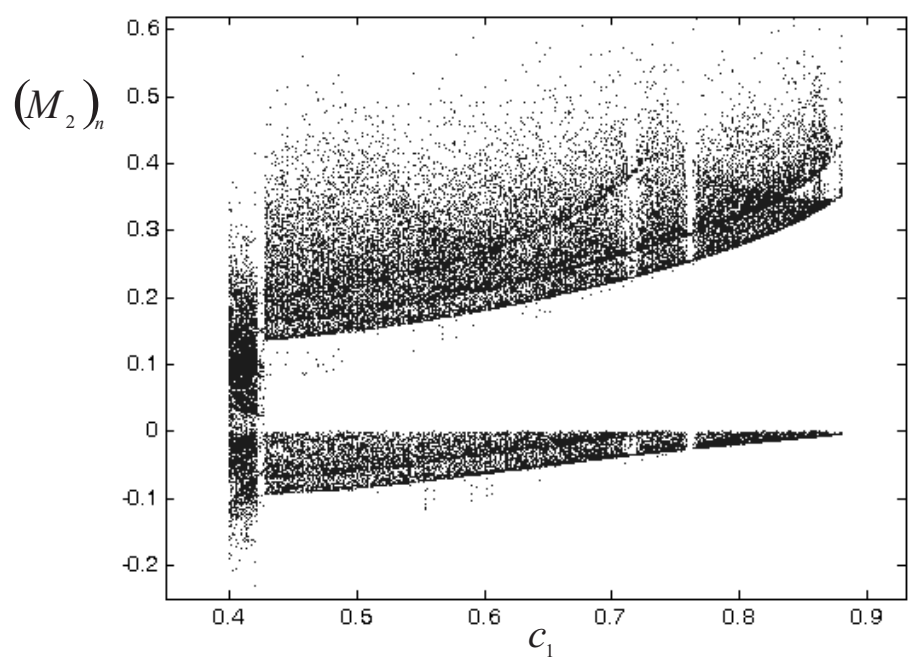

Fig. 5. Bifurcation diagram $\left(M_{2}\right)_{n}$ versus $c_{1}$, generated by computer solutions of the system (2), computed with the parameters: $c_{1} \in[0.4,0.88]$, $c_{3}=0.4, c_{2}=10, c_{4}=5, c_{5}=0.175, c_{6}=5$

two heteroclinic cycles $-\Gamma_{0}^{2,3} \subset W^{u(s)}\left(O_{2}\right) \cap W^{s(u)}\left(O_{3}\right) ; \Gamma_{0}^{4,5} \subset W^{u(s)}\left(O_{4}\right) \cap$ $W^{s(u)}\left(O_{5}\right)$, respectively - see Figs 8 and 9 in Appendix.

It is interesting, that in the second interval (see Fig. 6 right panel) the one strange attractor disappears/appears suddenly, as one heteroclinic cycle collapse.

\section{Summary and conclusions}

An important feature of robust heteroclinic cycles is that they may attract nearby dynamics. What happens, when a cycle loses stability? Such bifurcation may lead to the appearance of long period periodic orbits, other heteroclinic cycles, and more complicated dynamics.

The present paper studies numerically how the dynamics and global behaviour of system (2) vary, when we keep $c_{2}=10, c_{3} \in[0.4,0.9], c_{4}=5$, $c_{6}=5$ and change $c_{1}$ and $c_{5}$. We focused our estimations on the bifurcation behaviour, route to chaos and occurrence of homoclinic and heteroclinic structures (cycles). It is curious, that system (2), which is a purely mechanical interpretation of the Lorenz system, displays some more interesting, from dynamical point of view, behaviours. Our new results suggest that the system 

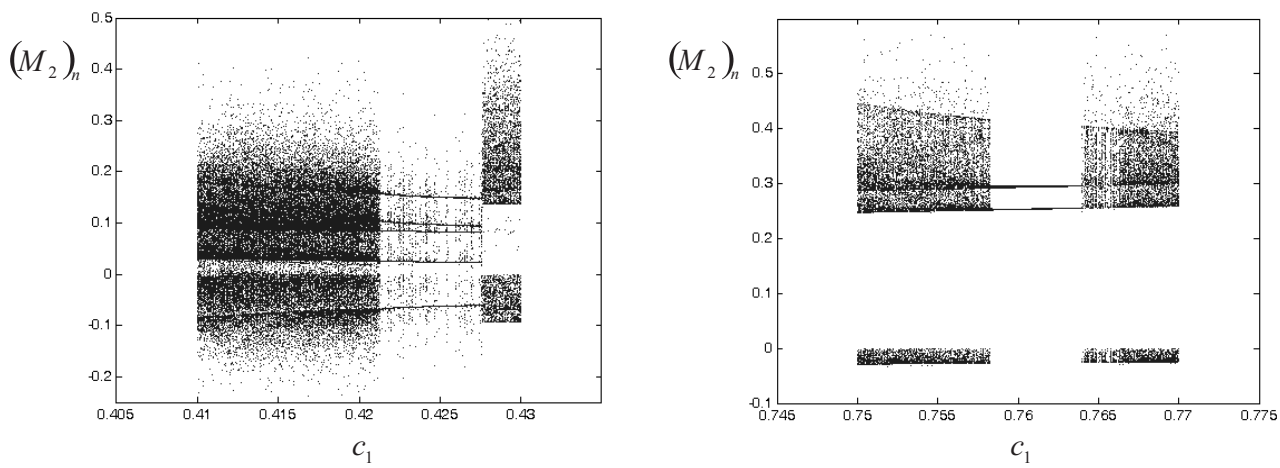

Fig. 6. Bifurcation diagrams of system (2), computed with the parameters: $c_{1} \in[0.41,0.43]-$ left panel and $c_{1} \in[0.75,0.77]$ - right panel

(2) possesses: (i) five unstable fixed points from saddle-focus type; (ii) a homoclinic structure for fixed point $O_{1}$; (iii) two heteroclinic structures $\Gamma_{0}^{2,3}$ and $\Gamma_{0}^{4,5}$, including two fixed points; (iv) homoclinic and heteroclinic orbits can exist at the same time. In the case, when the system possesses two strange attractors, two heteroclinic structures, including two fixed saddle-focuses take place, as for $c_{5} \in[0.758,0.763]$ the one strange attractor disappears/appears suddenly. In this case, the corresponding heteroclinic cycle collapses.

According to heteroclinic Shilnikov criteria, if system (11) has heteroclinic orbits of Shilnikov type, which connect two distinct saddle-foci of the system, then it has both Smale horseshoes and horseshoe type chaos.

In conclusion, concerning the system evolution and bifurcation scenarios, we find new results for its routes to chaos.

\section{REFERENCES}

[1] Li, I., J. Yorke. Period Three Implies Chaos. Amer. Math. Mountly, 82 (1975), 985-992.

[2] Ruelle, D., F. Takens. On the Nature of Turbulence. Comm. Math. Phys., 20 (1971), No. 2, 167-192.

[3] Idowu, B., U. Vincent, A. Nuah. Control and Synchronization of Chaos in Nonlinear Gyros via Backstepping Design. Int. J. Nonliner Sci., 5 (2008), No. 1, $11-19$.

[4] Firavar, F., M. Shoorehdeli, M. Nekoui, M. Teshnehlab. Chaos Control and Generalized Projective Synchronization of Heavy Symmetric Chaotic Gyro- 
scope Systems via Gaussian Radial Basis Adaptive Variable Structure Control. Chaos, Solitons 85 Fractals, 45 (2012), 80-97.

[5] Binhi, V., A. SAvin. Molecular Gyroscopes and Biological Effects of Weak Extremely Low-frequency Magnetic Fields. Physical Review E, 65 (2002), 051912 (10 pages).

[6] Leonhardt, U., P. Piwnicki. Ultrahigh Sensitivity of Slow-light Gyroscope. Physical Review A, 62 (2000), 055801 (2 pages).

[7] Will, C. Covariant Calculation of General Relativistic Effects in an Orbiting Gyroscope Experiment. Physical Review D, 67 (2003), 062003 (7 pages).

[8] Kalvouridis, T. Stationary Solutions of a Small Gyrostat in the Newtonian Field of Two Bodies with Equal Masses. Nonlinear Dynamics, 61 (2010), 373-381.

[9] Borisov, A., A. Kilin, I. Mamaev. Absolute and Relative Choreographies in Rigid Body Dynamics. Regular and Chaotic Dynamics, 13 (2008), No. 3, 204-222.

[10] Nikolov, S. Regular and Chaotic Behaviour of Fluid Gyroscope. Comptes rendus de l'Academie bulgare des Sciences, 57 (2004), No. 1, 19-26.

[11] Doroshin, A. Exact Solutions for Angular Motion of Coaxial Bodies and Attitude Dynamics of Gyrostat-satellites. Int. J. of Nonlinear Mechanics, 50 (2013), $68-74$.

[12] Nikolov, S., V. Kotev, E. Yankulova. Bifurcation and Dynamical Behaviour of a Mathematical Model of HIV Infection. J. of Theoretical and Applied Mechanics, 37 (2007), No. 2, 101-116.

[13] Islam, M., N. Islam, S. Nikolov. Adaptive Control and Synchronization of Sprott J System with Estimation of Fully Unknown Parameters. J. of Theoretical and Applied Mechanics, 45 (2015), No. 2, 43-56.

[14] Krupa, M. Robust Heteroclinic Cycles. J. Nonlinear Sci., 7 (1997), 129-176.

[15] Kuznecov, A., V. Afraimovich. Heteroclinic Cycles in the Repressilator Model. Chaos, Solitons \& Fractals, 45 (2012), No. 5, 660-665.

[16] Parthimos, D., D. Edwards, T. Griffith. Shilnikov Homoclinic Chaos is Intimately Related to Type- III Intermittency in Isolated Rabbit Arteries: Role of Nitric Oxide. Physical Review E, 67 (2003), 051922 (7 pages).

[17] Peiхото, M. Structural Stability on Two-dimensional Manifolds. Topology, 1 (1962), 101-120.

[18] Scott, S. Chemical Chaos. Oxford, Clarendon Press, 1991.

[19] Shilnikov, L. A Case of the Existence of a Denumerable Set of Periodic Motions. Sov. Math. Dokl., 6 (1965), 163-166.

[20] Wiggins, S. Global Bifurcations and Chaos: Analytical Methods, NY, SpringerVerlag, 1988.

[21] Wiggins, S. Introduction to Applied Nonlinear Dynamical Systems and Chaos, NY, Springer-Verlag, 1996.

[22] Lorenz, E. Deterministic Nonperiodic Flow. J. Atmos. Sci., 20 (1963), No. 2, 130-141.

[23] Gros, C. Complex and Adaptive Dynamical Systems: a Primer. Berlin, SpringerVerlag, 2008. 
[24] Neimark, Yu., P. Landa. Stochastic and Chaotic Oscillations. Dordrecht, Kluwer Academic Publishers, 1992.

[25] Tong, Ch. Lord Kelvin's Gyrostat and its Analogs in Physics, Including the Lorenz model. Am. J. Phys., 77 (2009), No. 6, 526-537.

[26] Gluhovsky, A. Nonlinear Systems that are Superpositions of Gyrostats. Sov. Phys. Dokl., 27 (1982), 823-825.

[27] Borisov, A., I. Mamaev. Rigid Body Dynamics. Moscow-Izhevsk, NIC, 2001 (in Russian).

[28] Leipnik, R., T. Newton. Double Strange Attractor in Rigid Body Motion with Linear Feedback Control. Phys. Lett., 86 (1981), No. 2, 63-67.

[29] Klein, F., A. Sommerfeld. Uber die Theorie des Kreiseds. New York, Johnson Reprint Corp, 1965.

[30] Bautin, N. Behaviour of Dynamical Systems Near Boundary of Stability. Moscow, Nauka, 1984 (in Russian).

[31] Homburg, A., B. Sandstede. Homoclinic and Heteroclinic Bifurcations in Vector Fields. In: Handbook of Dynamical Systems North-Holland, 3 (2010), 379-524.

[32] Gonchenko, S., D. Turaev, P. Gaspard, G. Nicolis. Complexity in the Bifurcation Structure of Homoclinic Loops to a Saddle-focus. Nonlinearity, 10 (1997), No. 2, 409.

[33] Dufraine, E., J. Danckaert. Some Topological Invariants for Threedimensional Flows. Chaos, 11 (2001), No. 3, 443-448.

[34] Guckenheimer, J., Ph. Holmes. Nonlinear Oscillations, Dynamical Systems, and Bifurcations of Vector Fields, New York, Springer, 1998.

[35] Gonchenko, S., I. Ovsyannikov. On Bifurcations of Three-dimensional Diffeomorphisms with a Non-transversal Heteroclinic Cycle Containing Saddle-foci. Nonlinear Dynamics, 6 (2010), No. 1, 61-77 (in Russian).

[36] Afraimovich, V., S. Gonchenko, L. Lerman, A. Shilnikov, D. Turaev. Scientific Heritage of L. P. Shilnikov. Regular and Chaotic Dynamics, 19 (2014), No. 4, 435-460.

[37] Shilnikov, L. On a New Type of Bifurcation of Multidimensional Dynamical Systems. Sov. Math., 10 (1969), 1368-1371.

[38] Kuznetsov, Yu. Elements of Applied Bifurcation Theory, 2 ed., New York, Springer, 1998.

[39] Newton, T., D. Martin, R. Leipnik. A Double Strange Attractor, In: Dynamical Systems Approaches to Nonlinear Problems in Systems and Circuits. SIAM (1988), 117-127.

[40] MATLAB, The MathWorks Inc., MA, Natick, 2010, http://mathworks.com/.

[41] Fan, Y., T. Chay. Crisis and Topological Entropy. Physical Review E, 51 (1995), 1012-1019.

[42] Alligod, K. T., T. D. Sauer, J. A. Yorke. Chaos. An Introduction to Dynamical Systems, New York, Springer-Verlag, 1996. 
Appendix

Additional figures
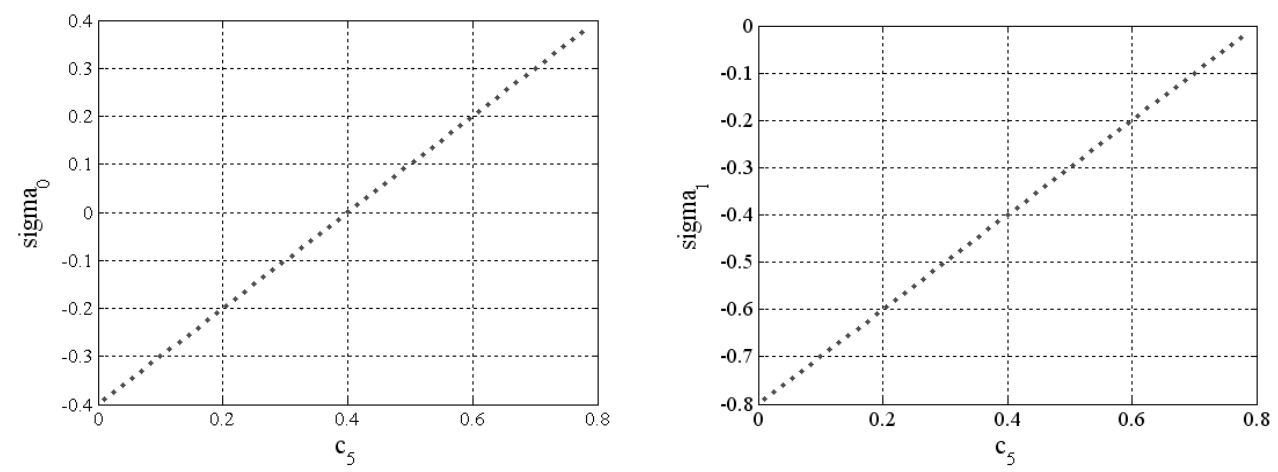

Fig. 7. Results for $\sigma_{0}$ and $\sigma_{1}$ of equilibrium $O_{1}$, as functions of control parameter $c_{5}$. According to Theorem, there are intervals where the basic cycle is stable
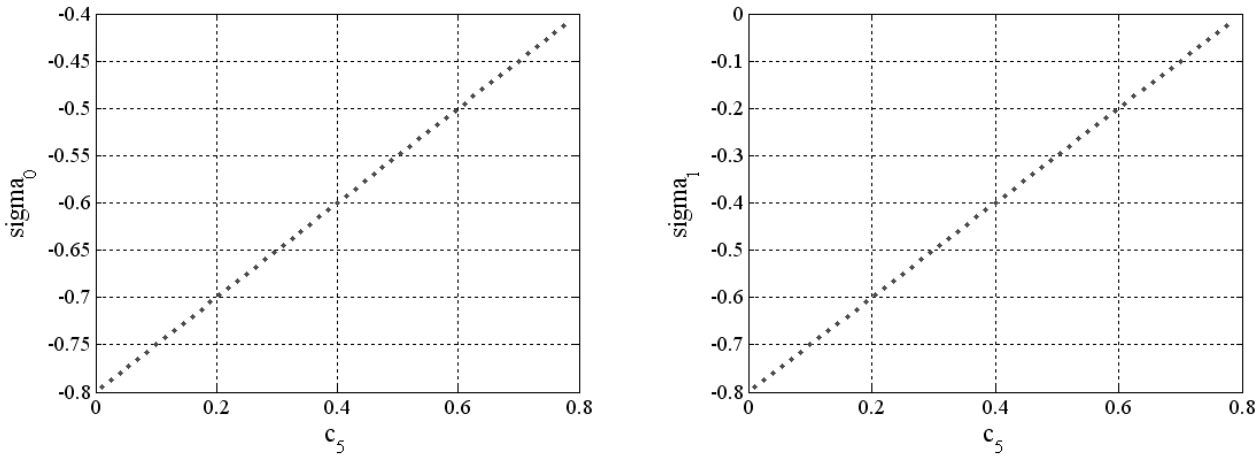

Fig. 8. Results for $\sigma_{0}$ and $\sigma_{1}$ of equilibrium $O_{2}$, as functions of control parameter $c_{5}$. According to Theorem, the system has infinite number of saddle limit cycles, and there are intervals, where the basic cycle is absolutely unstable (repelling). Here, we note that results for $O_{3}, O_{4}$ and $O_{5}$ are similar 


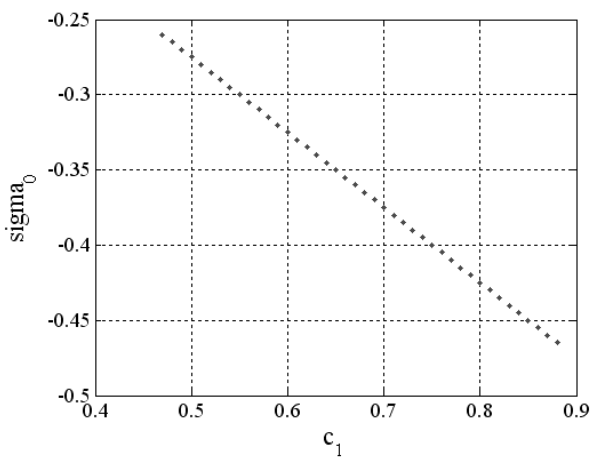

Fig. 9. Results for $\sigma_{0}$ of equilibrium $O_{1}$, as functions of control parameter $c_{1}$. According to Theorem, there are intervals where the one basic cycle is stable
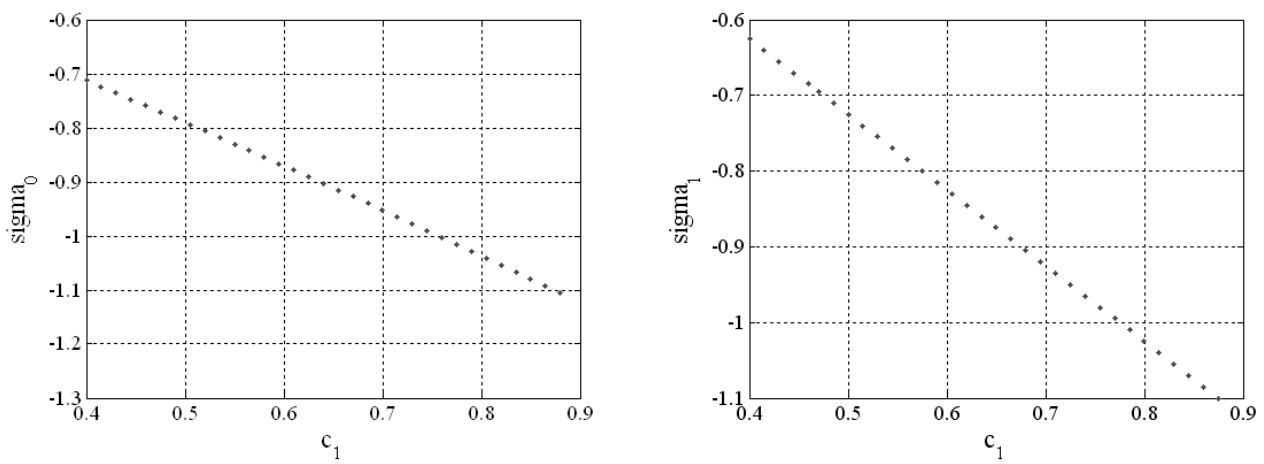

Fig. 10. Results for $\sigma_{0}$ and $\sigma_{1}$ of equilibrium $O_{2}$, as functions of control parameter $c_{1}$. According to Theorem, the system has infinite number of saddle limit cycles, and there are intervals where the basic cycle is absolutely unstable (repelling). Here, we note that results for $\mathrm{O}_{3}, \mathrm{O}_{4}$ and $\mathrm{O}_{5}$ are similar

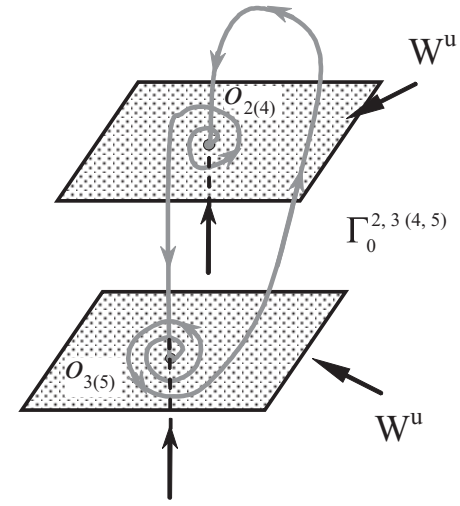

Fig. 11. Heteroclinic structure (cycle) from two saddle-foci 